\title{
ON THE CHOICE OF THICKNESS OF THE CEMENT MANTLE IN CEMENTED HIP ARTHROPLASTY
}

\author{
IEVGEN LEVADNYI \\ Lodz University of Technology, Department of Automation, Biomechanics and Mechatronics, Eódź, Poland \\ e-mail: evgenabaqus@gmail.com \\ JAN AWREJCEWICZ \\ Lodz University of Technology, Department of Automation, Biomechanics and Mechatronics, Eódź, Poland, and \\ Warsaw University of Technology, Institute of Vehicles, Warszawa, Poland; e-mail: jan.awrejcewicz@p.lodz.pl
}

\section{Alexander Loskutov}

Dnipropetrovsk State Medical Academy, Department of Orthopaedics and Traumatology, Dnipropetrovsk, Ukraine e-mail: loskutovae@ukr.net

\section{Olga Szymanowska}

Lodz University of Technology, Department of Automation, Biomechanics and Mechatronics, Lódź, Poland e-mail: olga.szymanowska@p.lodz.pl

\begin{abstract}
Recommendations for the optimal thickness of the cement mantle in cemented hip arthroplasty are outlined based on the results obtained with the finite element method. The investigations show that distal femur cement thickness higher than $2 \mathrm{~mm}$ positively affects mechanical behaviour of the cement mantle and can be useful in reducing stress-strain levels in the distal part of the femur what leads to prevention of development of a stress-shielding effect. The results of the study can contribute to the success of long-term implants.
\end{abstract}

Keywords: total hip replacement, bone cement, femur, stress state, finite elements method

\section{Introduction}

Nowadays, hip replacement is one of the most common and effective surgical methods for treatment of musculoskeletal system diseases and restoration of motion of the hip joint (Jenkins et al., 2013). Since 1959, by virtue of the work of Sir John Charnley (Charnley, 1960), numerous researchers working on the fixation in arthroplasty have used bone cement to fasten implants. Such a fixation of the prosthesis is advantageous in the case of a wide medullary canal of femur, osteoporosis, bone dysplasia, and consequences of mechanical injuries of hip and has been also traditionally used for femoral revision (Sullivan et al., 1994; Davis et al., 2003; Warth et al., 2014). Furthermore, cement fixation allows for a reduction of a rehabilitation period.

In the case of a cemented stem endoprosthesis of the hip joint, an important role is played by both strong bond between the implant surface and the bone cement as well as stable mechanical interaction between the cement and bone tissue. During the long-term use of the prosthesis, various difficulties, which can lead to aseptic loosening of the prosthesis and also to partial or complete loss of functionality of the whole system, occur (Huiskes, 1993; Bhambri and Gilbertson, 1995; Kroell et al., 2009). The above-mentioned conditions are usually observed in patients who have an overly active lifestyle or suffer from osteoporosis and/or overweight. They may also occur if the implant is incorrectly installed or if thickness of the cement mantle is wrongly chosen (Gunn et al., 2012), what results from low skills of a surgeon. 
It is of particular importance to properly choose thickness of the cement mantle while implanting a hip joint endoprosthesis, since too thin cement mantle may contribute to cracking of the cement, which, in turn, may lead to aseptic loosening of the implant. The complete absence of the mantle may lead to wear of bone (the so-called stress shielding effect) due to micro friction. On the other hand, too thick cement mantle will poorly transfer the load to the bone, which can lead to the bone atrophy and lysis. Thus, correctly chosen thickness of the cement mantle and proper positioning of the stem of the hip joint endoprosthesis in the medullary canal should ensure a long-term implant success.

However, the optimal thickness of the cement mantle has not been defined. Typically, its selection is the surgeon's responsibility and depends on the surgeon experience (Shah and Porter, 2005). In this regard, the development of a biomechanical rationale for the choice of the optimal cement mantle thickness in the cemented hip replacement is a compelling and urgent task, which will improve the outcome of treatment and increase durability of the prosthesis.

Currently, numerical simulation (especially the finite element method (FEM)) is one of the most effective and informative methods of research of problems related to biomechanics. By virtue of the FEM, it is possible to avoid difficulties associated with the use of analytical methods for calculation of the stress-strain state of biomechanical systems and, most importantly, to obtain high accuracy of results (Yamako et al., 2014).

The aim of this study is to analyse the stress state arising in a bone-cement-implant system with different sizes of tapered stems, depending on the thickness of the cement mantle. Such an analysis will contribute to finding the rationale for the size of the implant to avoid fatigue fracture of the cement and to provide even distribution of stress in the bone.

\section{Materials and methods}

For the analysis, a three-dimensional model of femur has been developed using computed tomography $(\mathrm{CT})$, which is a method allowing creation of highly accurate models of organs. CT provides information on the shape and properties of soft and bone tissue, giving almost an in vivo model. The algorithm of generation of a three-dimensional geometric model of the femur includes several steps. At first, CT images of the patient are downloaded for subsequent segmentation of the object. The segmentation is performed based on the obtained axial projections of the object, using the selection as a separate mask. Then, a STF file with the 3D object made on the mask is created. In the third step, the quality of the model is improved by employing various surface smoothing functions. Eventually, mechanical characteristics of femur are found by calculating analytical dependences between the Hounsfield units (HU) obtained from the computed tomograms (Fig. 1). Hounsfield units determine the dependence between radiographic density of the femur tissue, presented in arbitrary units (Cann, 1988; Peng et al., 2006), the actual bone density $\rho\left[\mathrm{g} / \mathrm{cm}^{3}\right]$, and the elastic modulus $E[\mathrm{MPa}]$ (Yosibash et al., 2007; Helgason et al., 2008; Khan et al., 2014). One can find numerous relationships between bone density and HU for different bone types. In our study, the relationship $\rho=1+7.185 \cdot 10^{-4} \cdot \mathrm{HU}$ (Peng et al., 2006; Laz et al., 2007; Snethen, 2013; Pérez et al., 2014) is used. Material properties of cortical and cancellous bone have been assigned according to the equations proposed by Wirtz et al. (2000)

$$
E[\mathrm{MPa}]=E(\rho)=\left\{\begin{array}{llll}
2065^{3.09} & \text { if } & \rho>1.2 \mathrm{~g} / \mathrm{cm}^{3} & \text { (cortical bone) } \\
1904^{1.64} & \text { if } & \rho \leqslant 1.2 \mathrm{~g} / \mathrm{cm}^{3} & \text { (cancellous bone) }
\end{array}\right.
$$

Poisson's ratio is assumed to be 0.3 for the whole analysed bone (Wirtz et al., 2000).

The model used in this work has the shape and size of an ORTAN ${ }^{\circledR}$ tapered femoral component (Ukraine). In general, such implants consist of two elements: a stem and a ball head (Fig. 2b). The stem comprises four parts, including cervical (2), head (1), metaphyseal (3), and 
(a)

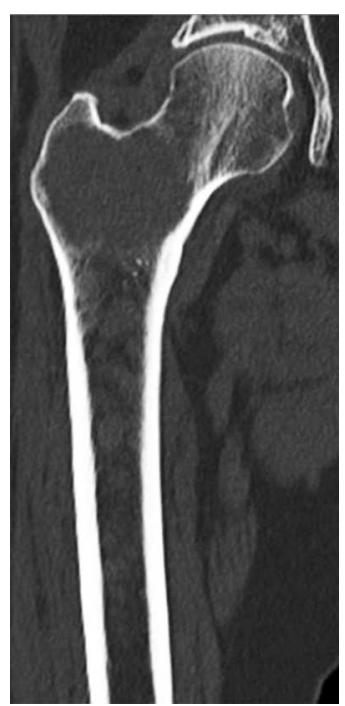

(b)

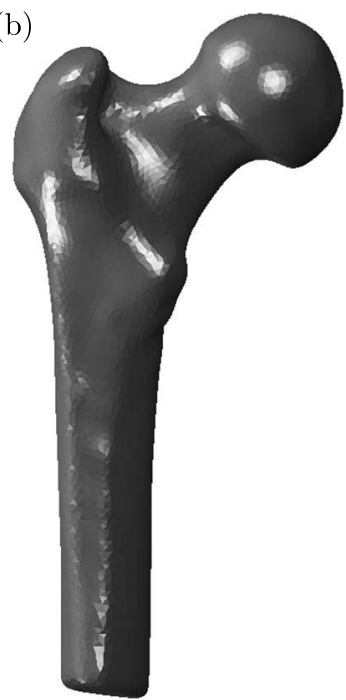

(c) Density, $\rho\left[\mathrm{g} / \mathrm{cm}^{3}\right]$

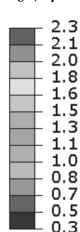

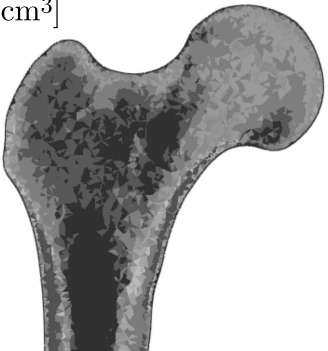

Elastic modulus, $E[\mathrm{MPa}]$

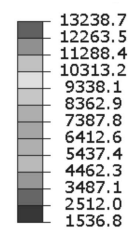

Fig. 1. Three-dimensional femur model created from CT images: (a) CT image, (b) solid geometry of the bone, (c) distribution of material properties: density $\rho\left[\mathrm{g} / \mathrm{cm}^{3}\right]$ and elastic modulus $E[\mathrm{MPa}]$ in the cross section of proximal femur

(a)
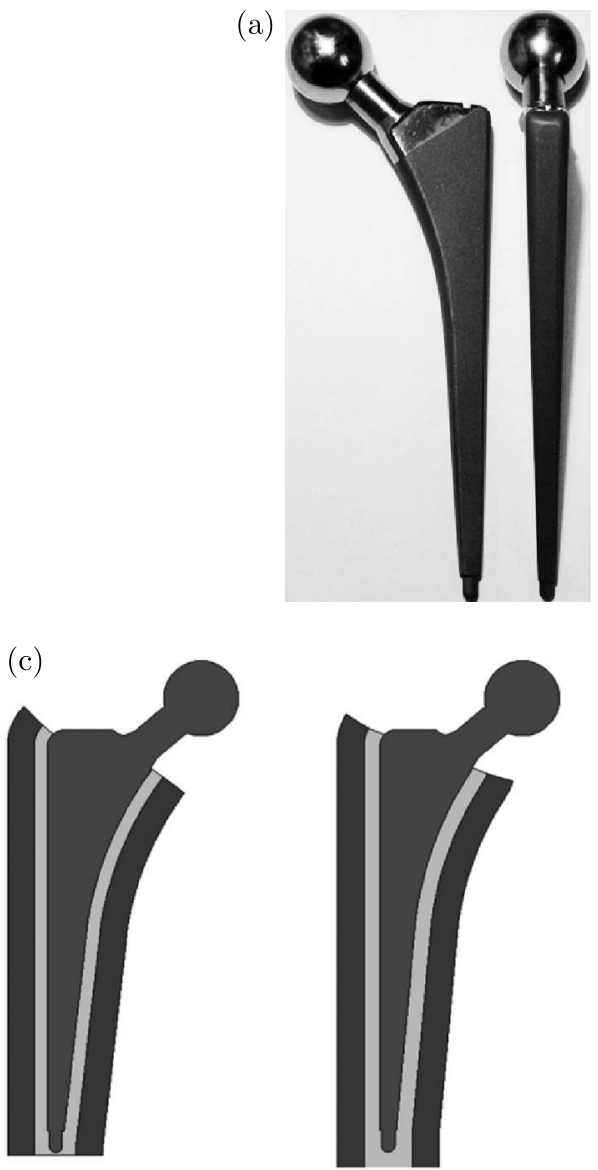

(b)

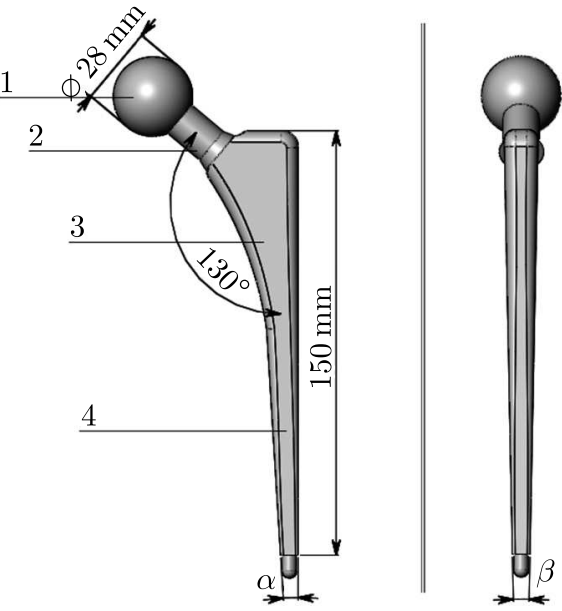

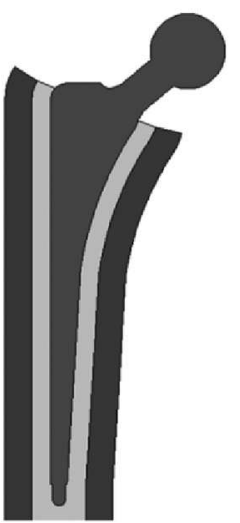
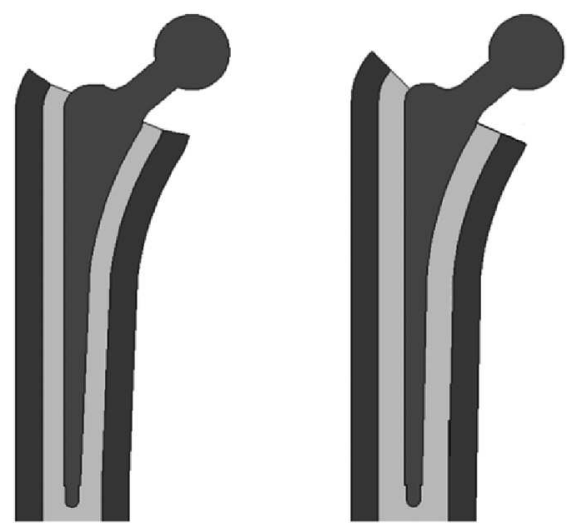

Fig. 2. Size 1 tapered stem hip endoprosthesis: (a) photograph of the implant; (b) 3D model used in numerical simulations ( 1 - head, 2 - cervical part at an angle of $130^{\circ}$ to the stem; 3 - metaphyseal part and 4 - diaphyseal part); (c) geometric model of cemented endoprosthesis with thickness of cement mantle (light grey) equal to 1, 2, 3, 4 and $5 \mathrm{~mm}$, respectively 
diaphyseal (4) ones. Cervical part (2), which is used to install head (1), has a form of a (Morse) cone. The stem of the prosthesis is wedge-shaped with an angle $\alpha$ to the sagittal plane and $\beta$ to the frontal plane. For this type of endoprosthesis, the cervico-diaphyseal angle is equal to $130^{\circ}$. The diameter of the head of the hip joint endoprosthesis is $28 \mathrm{~mm}$. Wedge-shaped metaphyseal part (3) contributes to stability and strength of fixation of the stem in the bone and resists subsidence of the stem prosthesis. Distal part of the stem (4) has a conical shape and is jammed in the front-rear direction. In the developed model, the femoral component has physical properties of stainless steel (316L) with Young's modulus $E=200 \mathrm{GPa}$ and Poisson's ratio 0.3.

In this paper, we consider several possible variations of installation of stems with different sizes in the bone cement that fills the medullary canal of femur. Owing to varying implant thickness, the thickness of the cement mantle is changed in a range between $1 \mathrm{~mm}$ and $5 \mathrm{~mm}$ (Fig. 2c). Young's modulus of the cement equals $2.5 \mathrm{GPa}$ and Poisson's ratio is 0.29. All contact surfaces of the cement mantle are assumed to be perfectly bonded to the surrounding bone. Contact between the implant and the cement is modelled using a surface-to-surface contact algorithm (in Abaqus software) with the Coulomb stick-slip model of friction with the friction coefficient equal to 0.25 , simulating the surface finish for the stem, consistent with the surface finish of the ORTAN ${ }^{\circledR}$ stems. The cement mantle is assumed to be fixed to the surrounding cortical and trabecular bone.
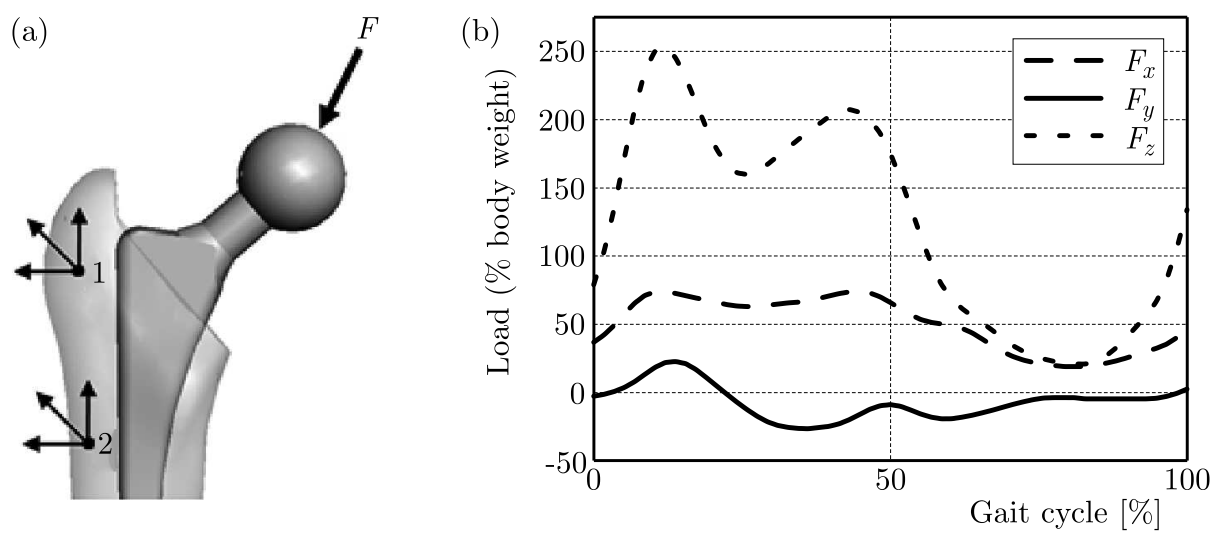

Fig. 3. Load cases: (a) hip contact force acting from the acetabular cup to the implant head of the magnitude $\mathbf{F}$ and its components $F_{x}$ (frontal axis), $F_{y}$ (sagittal axis) and $F_{z}$ (vertical axis); numbers 1 and 2 indicate points where the muscle forces act; (b) values of components $F_{x}, F_{y}$, and $F_{z}$ of the contact force $\mathbf{F}$ in percentage of the body weight $[\% \mathrm{BW}]$ during normal walking

Table 1. Physiological loading conditions on the lateral surface of femur during normal walking

\begin{tabular}{|l|c|c|c|c|}
\hline \multirow{2}{*}{ Muscle } & \multicolumn{3}{|c|}{ Load component [N] } & Initial \\
\cline { 2 - 4 } & $X$ & $Y$ & $Z$ & point \\
\hline \hline Abductor & 406 & 30.1 & 605.5 & 1 \\
\hline Tensor fascia lata, proximal part & 50.4 & 81.2 & 92.4 & 1 \\
\hline Tensor fascia lata, distal part & -3.5 & -4.9 & -133 & 1 \\
\hline Vastus lateralis & -6.3 & 129.5 & -650.3 & 2 \\
\hline
\end{tabular}

The contact load, acting from acetabulum to the head of the endoprosthesis, can be represented as a principal vector $\mathbf{F}$, which can be decomposed into three components acting along axes of the local Cartesian coordinate system associated with the centre of the prosthesis head. The force $\mathbf{F}$ and its components are presented in Fig. 3a. The dependence between $F_{x}, F_{y}$, and $F_{z}$ (expressed in percentage of the body weight) during a normal gait cycle is shown in Fig. 3b (Bergmann et al., 2001; Bergmann, 2008; Levadnyi et al., 2017). According to the plot, 
the maximum values of the components $F_{x}, F_{y}$, and $F_{z}$ are equal to: $F_{x}=0.743 Q, F_{y}=0.254 Q$, $F_{z}=2.649 Q$, respectively, where $Q$ is the weight of a human body (for body mass assumed to be $70 \mathrm{~kg}$ and the acceleration of gravity $9.81 \mathrm{~m} / \mathrm{s}^{2}$ ). In this case, the load acting on the femoral head equals: $F_{x}=510.2 \mathrm{~N}, F_{y}=174.4 \mathrm{~N}, F_{z}=1918.1 \mathrm{~N}$. In addition, the forces acting on the bone surface in muscle attachment sites have been also taken into account (Table 1) (Heller et al., 2005).

The finite element models of the bone-cement-implant system used in this study contain 1100123 3D solid four-node tetrahedral elements. For all elements of the system, length of the edge of mesh elements equals $1 \mathrm{~mm}$.

\section{Results}

In Figs. 4-6, the distributions and values of the equivalent von Mises stress in the bone-cement-hip implant system are reported for different thicknesses of the cement mantle and stem sizes. The curve number corresponds to thickness of the cement mantle in mm.

As shown, the stresses in bones are smaller if thinner stems are used, and bigger in the case of thicker implants. The results show that an increase in the stem thickness and the corresponding decrease in the cement mantle yield a stress increase in the distal part of femur. The maximum bone stresses are found on the medial side of femur, in the vicinity of the distal end of the stem. If the cement mantle thickness is equal to 1 to $2 \mathrm{~mm}$, there is a high concentration of stresses on the medial side of femur, in the contact zone of the distal stem and the cortical bone (Fig. 4). This can lead to development of the stress shielding effect and hypertrophy of femur. An increase

(a)

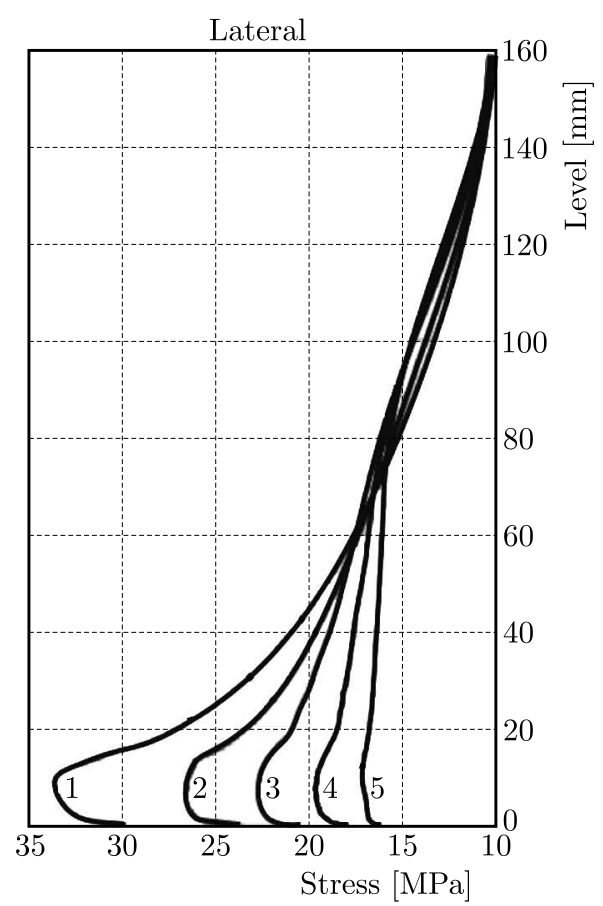

(b)

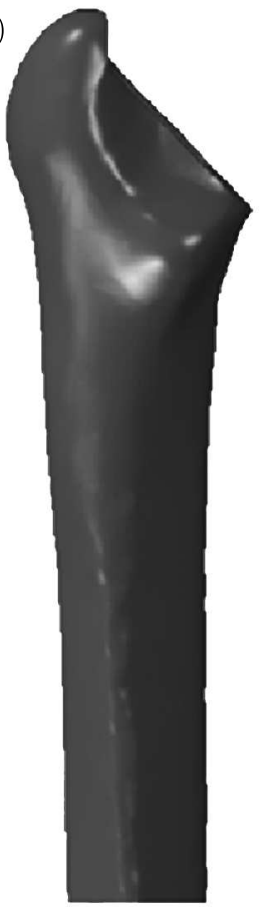

(c)

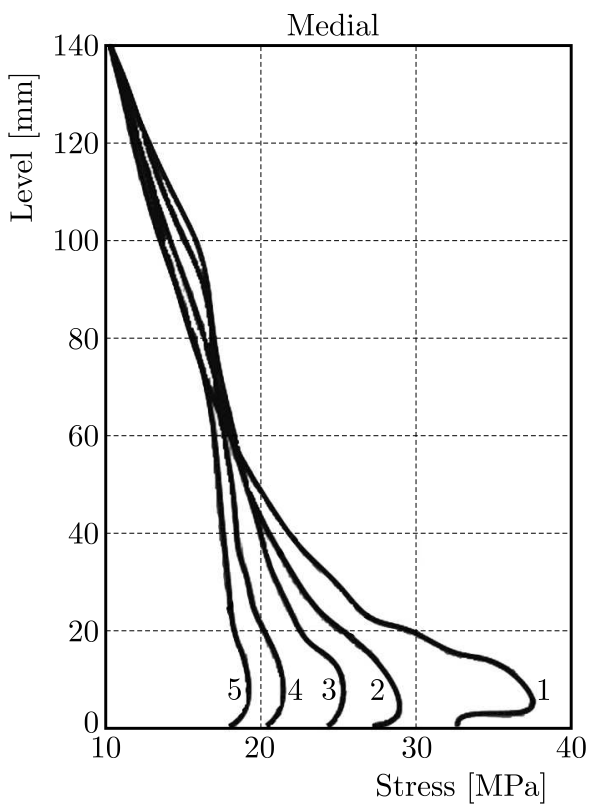

Fig. 4. (a) Distribution of the maximum equivalent stress on the lateral side of the femur depending on thickness of the cement mantle, (b) femur model, (c) distribution of the maximum equivalent stress on the medial side of the femur depending on thickness of the cement mantle

in thickness of the cement mantle causes a decrease in stresses in the distal part of the bone. If the thickness of the cement mantle ranges from 3.5 to $5 \mathrm{~mm}$, the stress values (tensile) on the 
lateral side and stress values (compression) on the medial side of the bone are evenly distributed excluding excessive stress concentration (Fig. 4).

For thickness of the cement mantle between 1 and $1.5 \mathrm{~mm}$, a peak stress is observed in the distal part. Its value exceeds the limit of durability of the bone cement (Fig. 5). In such a case, the cement mantle will not be able to ensure long-term functioning of the system, and hence there is a greater likelihood of loosening of the femoral component. According to the results, an increase in thickness of the cement mantle leads to reduction of stresses in the distal part of the cement mantle. Taking into account that, according to the manufacturers, the fatigue limit of bone cements is $8-10 \mathrm{MPa}$ (Kunh, 2000), a cement mantle thicker than $1.5 \mathrm{~mm}$ provides satisfactory conditions for functioning of the prosthesis under functional loads. Notice that while analysing the stress-strain state of the cement mantle, it is necessary to take into account stresses that occur on the lateral side (tensile stresses), since mechanical properties of the cement in tension are 9 times lower than those of the cement in compression.

(a)

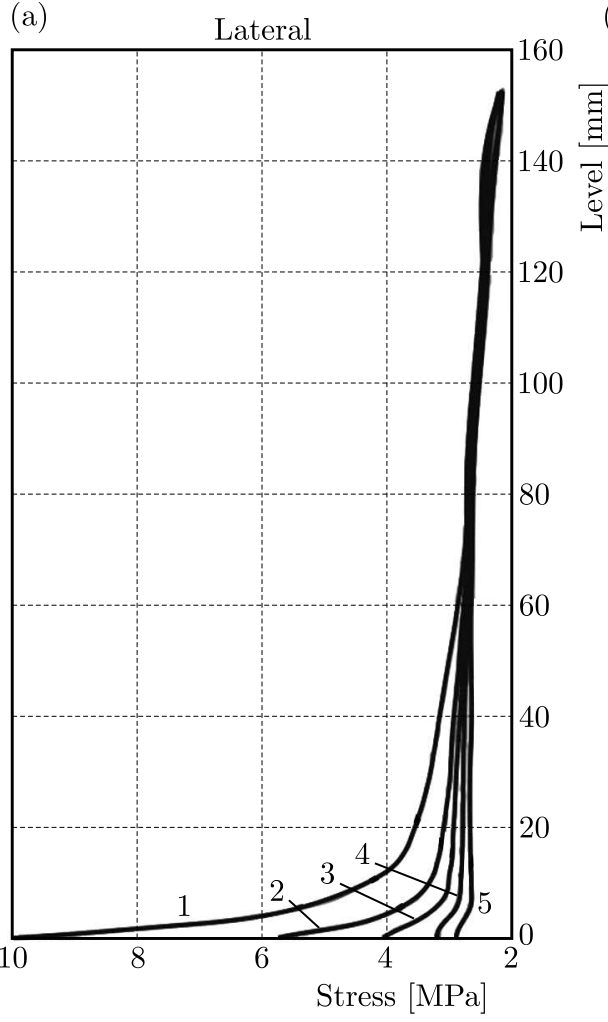

(b)

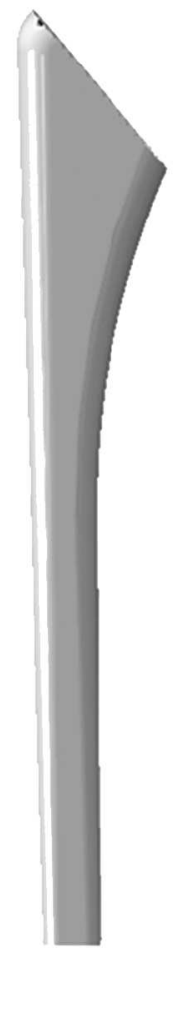

(c)

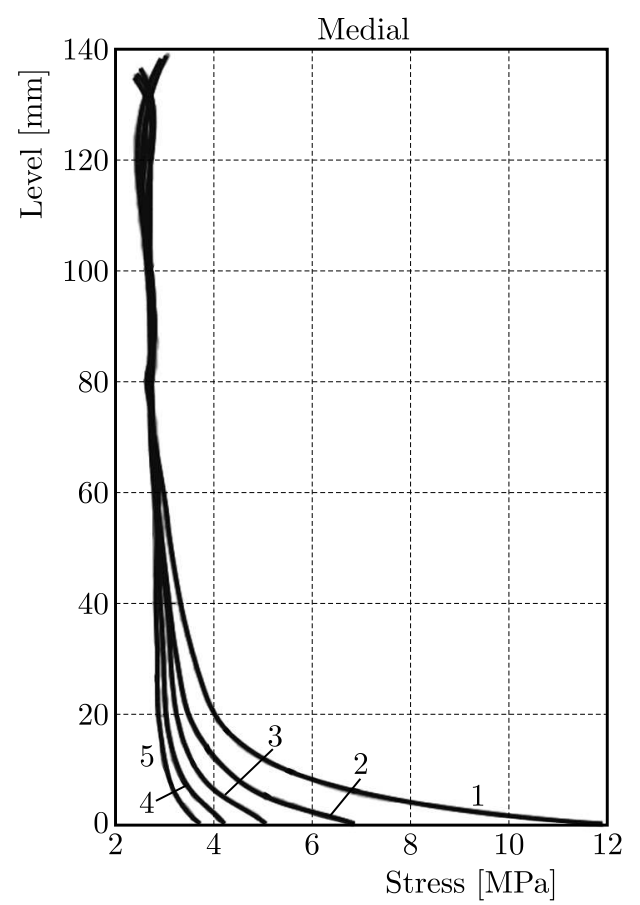

Fig. 5. (a) distribution of the maximum equivalent stress on the lateral side of the cement mantle depending on its thickness, (b) cement mantle model, (c) distribution of the maximum equivalent stress on the medial side of the cement mantle depending on its thickness

The stress-strain state of the stem is determined by a combination of the bending moment acting in the frontal plane and compression forces acting in the axial direction. The results show that the maximum stresses in the implant are found on the medial side, in the vicinity of the distal end of the stem of the prosthesis (Fig. 6). In general, the calculation results indicate that, regardless of the size of the endoprosthesis, the maximum implant stress does not exceed the yield strength of the material, and all implants are in the state of elastic deformation. This means that stresses in the material of implants under the functional loads are not dangerous to the structure of the implant. In this case, the dangerous stress value is assumed to be greater than the fatigue strength of 316L stainless steel, which is usually used to manufacture implants, this is greater than about $250 \mathrm{MPa}$ (Maruyama et al., 2011). Thus, the use of these structures provides a sufficient safety margin. However, the above observations are valid only for correct 
implantation of the prosthesis in femur. In the case of incorrect installation or presence of defects, pores, cracks, gaps, or distortions in the bone, implant or cement mantle, an increase in the stresses near the concentrator is observed, which causes fatigue failure of components or loss of stability of the system.
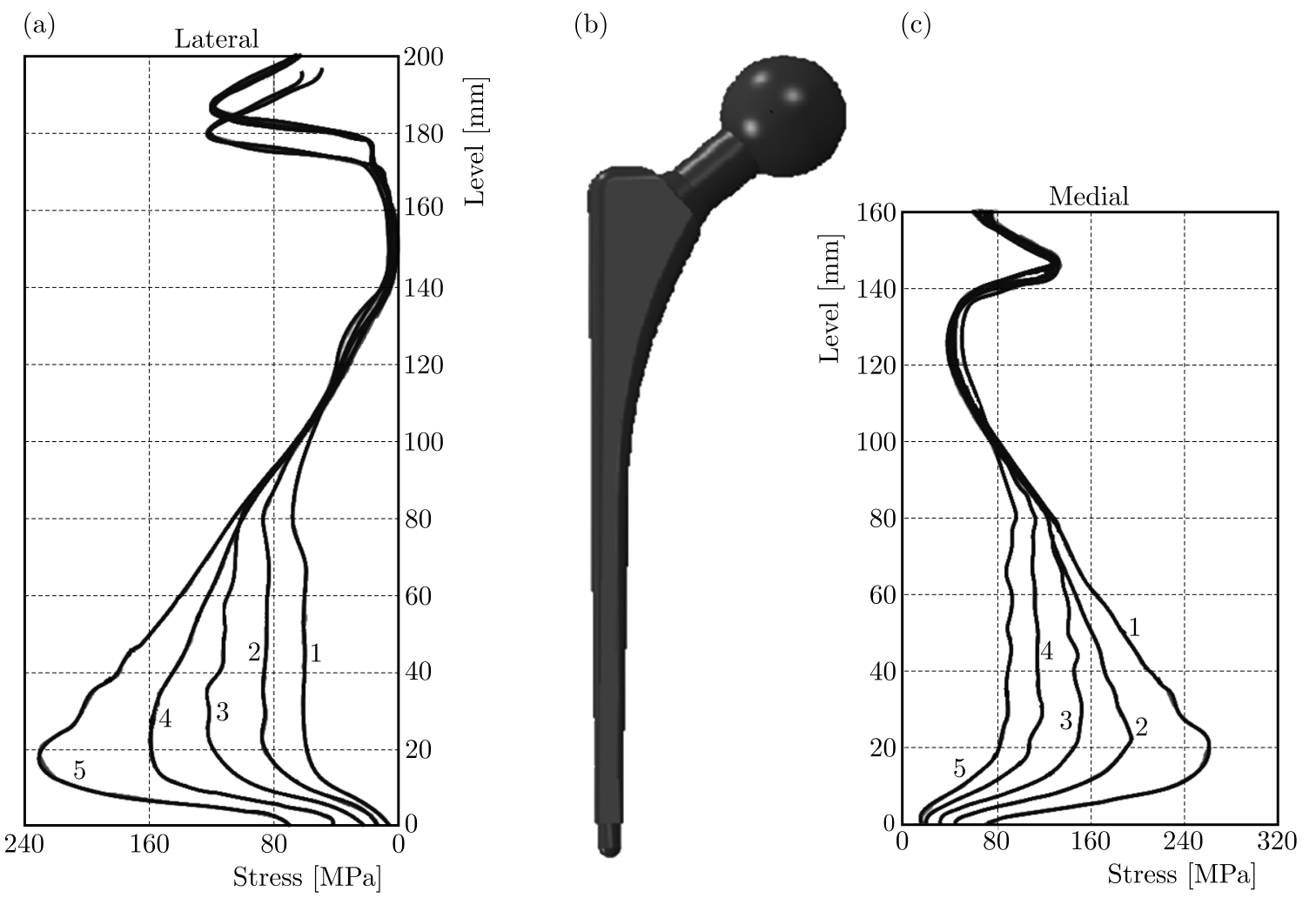

Fig. 6. (a) distribution of the maximum equivalent stress on the lateral side of the stem depending on thickness of the cement mantle, (b) model of the stem, (c) distribution of the maximum equivalent stress on the medial side of the stem depending on thickness of the cement mantle

\section{Discussion}

Improved cementing techniques and stem positioning are considered to be crucial factors for a long-term implant success. One of the methods used by researchers aimed at improving the orthopedic clinical practice is the finite element method, which is also applied in the present study.

For all considered thicknesses of the cement mantle under the action of functional loads, intensity and distribution of stresses have been obtained for both medial and lateral sides. The stress-strain state of the stem, cement mantle, and femur have been determined by combining the bending moment acting in the frontal plane and compression forces acting in the axial direction. As described in Section 3, the longitudinal tensile stress acts on the lateral side and the cervical part of the stem, cement mantle and bone, while compressive stresses are present on the medial side. The values of tensile stress in the cement mantle and femur are smaller than the values of the compressive stress.

The results have shown that changes in the cement mantle thickness around cemented femoral components can lead to significant changes in the stress-strain state of bone-cement-femoral component systems. For instance, an increase in the cement mantle thickness from 2 to $5 \mathrm{~mm}$ has led to a $47 \%$ reduction in stress in the distal medial strain gage and a $50 \%$ reduction in 
the distal lateral cement stress. This data is an experimental verification of the results from finite-element modeling reported by Estok et al. (1991), in which a 45-55\% reduction in peak distal cement strains was obtained by increasing cement mantle thickness from 2.5 to $5 \mathrm{~mm}$ and keeping the prosthesis diameter constant. A subsequent computational study by Lee et al. (1994) showed a reduction in peak tensile cement stresses by $45 \%$ when the cement mantle thickness was increased from 2 to $5 \mathrm{~mm}$ by means of reducing the prosthesis diameter. Also Fisher et al. (1997) conducted an experiment with two different sizes of cobalt-chromium stems and proved that in the case of increasing the cement mantle thickness from 2.4 to $3.7 \mathrm{~mm}$, substantial strain reductions in the distal cement (40-49\%) were observed.

In our work, the numerical simulation results have also shown that thickness of the cement mantle affects only the distal part of the cement mantle and does not affect the proximal one. Thus, one may conclude that the cement mantle should not be of the same thickness throughout the medullary canal of femur. Instead, to provide satisfactory conditions of functioning of the prosthesis under action of functional loads, the cement mantle should not be thicker than $1 \mathrm{~mm}$ in the proximal part of the femur. At the same time, most of the cement should be located in the distal part. The above-mentioned conditions should be ensured: 1) satisfactory conditions of functioning of the prosthesis, 2) even distribution of stresses in the bone (stresses concentration can be avoided). According to outcomes of this study, a slightly thicker cement mantle (up to $3 \mathrm{~mm}$ ) can be useful in reducing stress-strain levels around cemented femoral components. To thicken the cement mantle, either a smaller stem can be used or the femoral medullary canal can be expanded in the distal femur.

\section{Conclusions}

To obtain reliable results of the finite element analysis, models of both femur and endoprosthesis have been generated. The size, shape, material physicomechanical properties, and values of physical load of the models obtained for numerical simulations are the same as of real objects.

It has been found that, since all system elements work under cyclic load, the stress analysis should be conducted for comparison of the occurred stresses in the system components and the limit durability of the material from which the elements are made of. It has been also detected that when the bone cement in distal parts is thicker than $3 \mathrm{~mm}$, the load acting on tissue in the distal part of femur is significantly reduced. On the other hand, in the proximal part of femur, thickness of the cement mantle should not be smaller than $1.5 \mathrm{~mm}$ to ensure satisfactory conditions for functioning of the prosthesis under action of functional loads. What should be also emphasized, stresses in the stem of the endoprosthesis, regardless of thickness of the cement mantle, are lower than the fatigue strength of 316L steel used in hip implants, and thus fatigue fractures of stems are unlikely to occur.

\section{Acknowledgment}

The work has been supported by the National Science Centre of Poland under the grant OPUS 9 No. 2015/17/B/ST8/01700 for years 2016-2018.

\section{References}

1. Bergmann G., Deuretzbacher G., Heller M., Graichen F., Rohlmann A., Strauss J., Duda G.N., 2001, Hip contact forces and gait patterns from routine activities, Journal of Biomechanics, 34, 7, 859-871

2. Bergmann G. (ED.), 2008, OrthoLoad database, Charité-Universitätsmedizin Berlin, www.OrthoLoad.com, Accessed on December 10, 2016 
3. Bhambri S.K., Gilbertson L.N., 1995, Micromechanisms of fatigue crack initiation and propagation in bone cements, Journal of Biomedical Materials Research, 29, 2, 233-237

4. CAnn, C., 1998, Quantitative CT for determination of bone mineral density: a review, Journal of Radiology, 166, 509-522

5. Charnley J., 1960, Surgery of the hip joint, British Medical Journal, 1, 821-826

6. Devis C.M., Berry D.J., Harmsen W.S., 2003, Cemented revision of failed uncemented femoral components of total hip arthroplasty, The Journal of Bone and Joint Surgery, 85-A, 7, 1264-1269

7. Estok D.M., Harrigan T.P., Harris W.H., 1991, Finite element analysis of cement strains at the tip of an idealized cemented femoral component, Transactions of Orthopaedic Research Society, 16, 504

8. Fisher D.A., Tsang A.C., Paydar N., Milionis S., Turner C.H., 1997, Cement-mantle thickness affects cement strains in total hip replacement, Journal of Biomechanics, 30, 11-12, $1173-1177$

9. Gunn E, Gundapaneni D, Goswami T., 2012, Effect of cement fill ratio in loosening of hip implants, Biomatter, 2, 2, 87-93

10. Helgason B. Perilli E. Schileo E. Taddei F. Brynjolfsson S. Viceconti M., 2008, Mathematical relationships between bone density and mechanical properties: a literature review, Journal of Clinical Biomechanics, 23, 1, 135-146

11. Heller M.O., Bergmann G., Kassi J.-P., Claes L., Haas N.P., Duda G.N., 2005, Determination of muscle loading at the hip joint for use in pre-clinical testing, Journal of Biomechanics, 38, $5,1155-1163$

12. Huiskes R., 1993, Mechanical failure in total hip arthroplasty with cement, The Journal of Current Orthopaedics, 7, 239-247

13. Jenkins P.J., Clement N.D., Hamilton D.F., Gaston P., Patton J.T., Howie C.R., 2013, Predicting the cost-effectiveness of total hip and knee replacement: a health economic analysis, The Bone and Joint Journal, 95-B, 115-121

14. Khan S.N., Warkhedkar R.M., Shyam A.K., 2014, Analysis of Hounsfield unit of human bones for strength evaluation, Procedia Materials Science, 6 (2014), 512-519

15. Kroell A., Beaulé P., Krismer M., Behensky H., Stoeckl B., Biedermann R., 2009, Aseptic stem loosening in primary THA: migration analysis of cemented and cementless fixation, Journal of International Orthopaedics, 33, 6, 1501-1505

16. Kunn K., 2000, Bone Cements: Up-To-Date Comparison of Physical and Chemical Properties of Commercial Materials, Springer, Berlin

17. Laz P.J., Stowe J.Q., Baldwin M.A., Petrella A.J., Rullkoetter P.J., 2007, Incorporating uncertainty in mechanical properties for finite element-based evaluation of bone mechanics, Journal of Biomechanics, 40, 2831-2836

18. Lee I.Y., Skinner H.B., Keyak J.H., 1994, Effects of variation of prosthesis size on cement stress at the tip of a femoral implant, Journal of Biomedical Materials Research, 28, 1055-1066

19. Levadnyi I., Awrejcewicz J., Fagundes-Goethel M., Loskutov A., 2017, Influence of the fixation region of a press-fit hip endoprosthesis on the stress-strain state of the "bone-implant" system, Computers in Biology and Medicine, 84, 195-204

20. Maruyama N., Mori D., Hiromoto S., Kanazawa K., Nakamura M., 2011, Fatigue strength of 316L-type stainless steel in simulated body fluids, Journal of Corrosion Science, 53, 2222-2227

21. Peng L., Bai J., Zeng X., Zhou Y., 2006, Comparison of isotropic and orthotropic material property assignments on femoral finite element models under two loading conditions, Journal of Medical Engineering and Physics, 28, 3, 227-233 
22. PÉrez M.A., Vendittoli P.-A., Lavigne M., Nuño N., 2014, Bone remodeling in the resurfaced femoral head: Effect of cement mantle thickness and interface characteristics, Medical Engineering and Physics, 36, 2, 185-195

23. Shah N., Porter M., 2005, Evolution of cemented stems, Journal of Orthopedics, 28, 8, 819-825

24. Snethen K., 2013, A computed tomography-based model of the infant hip anatomy for dynamic finite element analysis of hip dysplasia biomechanics, HIM 1990-2015, 1465

25. Sullivan P.M., Mackenzie J.R., Callaghan J.J., Johnston R.C., 1994, Total hip arthroplasty with cement in patients who are less than fifty years old. A sixteen to twenty-two-year follow-up study, The Journal of Bone and Joint Surgery, 76, 6, 863-869

26. Warth L.C., Callaghan J.J., Liu S.S., Klaassen A.L., Goetz D.D., Johnston R.C., 2014, Thirty-five-year results after Charnley total hip arthroplasty in patients less than fifty years old. A concise follow-up of previous reports, The Journal of Bone and Joint Surgery. American Volume, 96, 21, 1814-1819

27. Wirtz D.C., Schiffers N., Pandorf T., Radermacher K., Weichert D., Forst R., 2000, Critical evaluation of known bone material properties to realize anisotropic FE-simulation of the proximal femur, Journal of Biomechanics, 33, 10, 1325-1330

28. Yamako G., Chosa E., Zhao X., Totoribe K., Watanabe S., Sakamoto T., Nakane N., 2014, Load-transfer analysis after insertion of cementless anatomical femoral stem using pre- and post-operative CT images based patient - specific finite element analysis, Medical Engineering and Physics, 36, 694-700

29. Yosibash Z., Trabelsi N., Milgrom C., 2007, Reliable simulations of the human proximal femur by high-order finite element analysis validated by experimental observations, Journal of Biomechanics, 40, 16, 3688-3699

Manuscript received February 6, 2017; accepted for print May 10, 2017 